\title{
Intraradial administration of fasudil inhibits augmented Rho kinase activity to effectively dilate the spastic radial artery during coronary artery bypass grafting surgery
}

\author{
Takeshi Takagi, MD, ${ }^{\text {a }}$ Yasuo Okamoto, MD, PhD, ${ }^{\mathrm{b}}$ Shigeyuki Tomita, MD, PhD, ${ }^{\mathrm{a}}$ Atsushi Sato, MMSc, \\ Shohjiro Yamaguchi, MD, PhD, ${ }^{\mathrm{a}}$ Yoh Takuwa, $\mathrm{MD}, \mathrm{PhD},{ }^{\mathrm{b}}$ and Go Watanabe, $\mathrm{MD}, \mathrm{PhD}^{\mathrm{a}}$
}

\begin{abstract}
Objectives: Radial arteries are increasingly used as conduits for coronary artery bypass grafts. However, vasospasm continues to be a major concern in radial artery grafts. Rho kinase plays a critical role in vascular contraction through phosphorylation of the regulatory subunit myosin phosphatase targeting subunit 1 (MYPT1) of the myosin light chain phosphatase to inhibit myosin light chain phosphatase in vascular smooth muscle. The purpose of this study was to evaluate the inhibitory effects of fasudil, a clinically used Rho kinase inhibitor, on Rho kinase activity, myosin light chain phosphorylation, in vitro contraction, and in situ vasospasm in radial arteries of patients undergoing coronary artery bypass grafting surgery.
\end{abstract}

Methods: The inhibitory efficacy of fasudil on vasoconstrictor-induced contraction and phosphorylation of MYPT1 was examined in radial artery rings. In situ phosphorylation of MYPT1 was evaluated in nonspastic and spastic radial arteries, and the effects of intraluminal administration of fasudil and verapamil-glyceryl trinitrate (VG) on in situ free blood flow and phosphorylation of MYPT1 and myosin light chain were compared in spastic radial arteries.

Results: Both fasudil and VG nearly fully inhibited noradrenaline- and serotonin-induced contraction of radial artery rings. However, fasudil but not VG abolished MYPT1 phosphorylation. In spastic radial arteries phosphorylation of MYPT1 and myosin light chain was increased compared with that seen in nonspastic arteries. Intraradial administration of fasudil induced a much larger increase in in situ free blood flow compared with VG treatment. This antispastic effect of fasudil was accompanied by marked decreases in phosphorylation of MYPT1 and myosin light chain.

Conclusions: Fasudil is a very effective Rho kinase inhibitor that deinhibits myosin light chain phosphatase and powerfully relieves vasospasm in situ in radial arteries. (J Thorac Cardiovasc Surg 2011;142:e59-65)

As an important prognostic factor of coronary artery bypass grafting $(\mathrm{CABG})$, graft spasm increased the incidence of angina and myocardial infarction during the perioperative period. ${ }^{1}$ The radial artery (RA) and the great saphenous vein are commonly used as composite grafts with the internal thoracic artery. ${ }^{1-4}$ However, the RA is more prone to spasm than the internal thoracic artery and great saphenous vein, probably because of the spastic property of the thick muscular wall and enhanced reactivity to vasoactive mediators. ${ }^{1,5,6}$ Therefore improvement in harvesting techniques and antispastic agents has been the subject of intense investigation. ${ }^{1,5}$ A number of drugs have been used

\footnotetext{
From the Departments of General and Cardiothoracic Surgery ${ }^{\mathrm{a}}$ and Physiology, ${ }^{\mathrm{b}}$ Kanazawa University Graduate School of Medicine, Kanazawa, Japan

Disclosures: Authors have nothing to disclose with regard to commercial support.

Received for publication Oct 14, 2010; revisions received Jan 10, 2011; accepted for publication Jan 25, 2011; available ahead of print March 14, 2011.

Address for reprints: Takeshi Takagi, MD, Kanazawa University Graduate School of Medicine, 13-1 Takara-machi, Kanazawa, Ishikawa 920-8640, Japan (E-mail: randel@kinbyou.hosp.go.jp).

$0022-5223 / \$ 36.00$

Copyright (C) 2011 by The American Association for Thoracic Surgery

doi:10.1016/j.jtcvs.2011.01.055
}

to prevent arterial graft spasm, which include a verapamilglyceryl trinitrate $(\mathrm{VG})$ solution $^{6-8}$; subtype-nonspecific and specific phosphodiesterase inhibitors, such as papaverine and olprinone ${ }^{6,9-11}$; and $\alpha$-adrenergic blockers. ${ }^{11}$ Among these antispasmodic agents, VG solution is considered to represent a relatively superior antispastic agent. ${ }^{6-8}$ Despite the use of these antispasmodics, RA vasospasm remains a problem that causes early graft failure after $\mathrm{CABG} .{ }^{1,5,6}$ Therefore the most effective way to prevent and reverse RA spasm is still under investigation.

Augmented smooth muscle contractility is thought to be a major cause of vasospasm that is induced by various stimuli, including mechanical trauma and vasoactive mediators. ${ }^{1,5,6}$ However, a detailed mechanism of vasospasm still remains unclear. An increase in the intracelluar $\mathrm{Ca}^{2+}$ concentration by contractile stimuli triggers phosphorylation of the 20-kd myosin light chain (MLC) through activation of $\mathrm{Ca}^{2+}$ / calmodulin-activated MLC kinase and initiates smooth muscle contraction. ${ }^{12,13}$ Vasoactive receptor agonists also activate the small guanosine triphosphatase Rho and its effector, Rho kinase (ROCK), leading to inhibition of the MLC-dephosphorylating enzyme MLC phosphatase (MLCP), which acts to potentiate MLC kinase-catalyzed 


$$
\begin{aligned}
& \text { Abbreviation and Acronyms } \\
& \mathrm{CABG}=\text { coronary artery bypass grafting } \\
& \text { 5-HT =5-hydroxytryptamine } \\
& \text { MLC = myosin light chain } \\
& \text { MLCP = myosin light chain phosphatase } \\
& \text { MYPT1 }=\text { myosin phosphatase targeting } \\
& \text { subunit } 1 \\
& \text { NA }=\text { noradrenalin } \\
& \mathrm{RA}=\text { radial artery } \\
& \text { ROCK }=\text { Rho kinase } \\
& \mathrm{VG}=\text { verapamil-glyceryl trinitrate }
\end{aligned}
$$

MLC phosphorylation and contraction in vascular smooth muscle. ${ }^{12,13}$ ROCK inhibits MLCP through phosphorylating the 110-kd myosin-targeting subunit myosin phosphatase targeting subunit 1 (MYPT1) of MLCP. ROCK-dependent MLCP inhibition is implicated in pathological vascular hypercontractile conditions; clinical trials have demonstrated that fasudil, a ROCK inhibitor, is effective in suppressing vasospasm after subarachnoid hemorrhage and coronary artery spasm. ${ }^{14-18}$

Recently, it has been reported that ROCK inhibitors, such as fasudil and Y27632, prevent agonist-induced vasoconstriction in isolated human RAs in vitro ${ }^{19,20}$ and that the mRNA and protein expression of Rho and ROCK in the RA was increased compared with that seen in the internal thoracic artery. ${ }^{21}$ In the present study we first examined the efficacy of fasudil on vasoactive agonistinduced contraction and MLCP inhibition in isolated RA rings. We then studied whether skeletonization-induced in situ spasm of RA was accompanied by augmented ROCK-mediated inhibition of MLCP and tested the reversing effects of intraradial fasudil application on spasm and MLCP inhibition in RAs of patients undergoing CABG. We demonstrate for the first time that intraradial application of fasudil in the spastic RA effectively increases in situ free blood flow with reversal of MLCP inhibition.

\section{MATERIALS AND METHODS Materials}

The following reagents were used in the present study: 5-hydroxytryptamine (5-HT; serotonin), noradrenalin (NA), papaverine, and mouse monoclonal anti-MLC antibody (M4401; Sigma, St Louis, Mo); dithiothreitol, trichloroacetic acid, and acetone (Wako Pure Chemicals, Osaka, Japan); rabbit polyclonal anti-phospho $\left(\mathrm{Thr}^{853}\right)$-MYPT1 (36-003) antibody (Upstate Biotechnology, Lake Placid, NY); rabbit polyclonal anti-MYPT1 antibody (PRB-457C; Covance, Berkley, Calif); rabbit polyclonal antiphospho (Ser $\left.{ }^{19}\right)$-MLC (3675) antibody (Cell Signaling, Danvers, Mass); fasudil (HA-1077; Asahi Chemical Industry, Fuji, Japan); verapamil and olprinone (Eizai Pharm, Inc, Tokyo, Japan); and glyceryl trinitrate (nitroglycerin; Astellas Pharma, Inc, Tokyo, Japan).

\section{Collection of RAs and Measurement of In Situ Free Blood Flow}

RA specimens were collected from 26 patients (19 male and 7 female patients) who underwent CABG with an RA graft at Kanazawa University Hospital. The procedures and handling of human tissue were approved by the ethical committee of Kanazawa University, and informed consent was obtained from each patient before the study. The mean age of the patients was $67.4 \pm 0.6$ years. Among 26 patients, 16 had hypertension, 11 had diabetes mellitus, and 12 had hyperlipidemia. The patients received medications of coronary vasodilators, including nitrate $(\mathrm{n}=12), \mathrm{Ca}^{2+}$ antagonists $(\mathrm{n}=13)$, and nicorandil $(\mathrm{n}=14)$; antiplatelet agents, including aspirin $(\mathrm{n}=22)$ and clopidogrel $(\mathrm{n}=7 ; \beta$-blockers $(\mathrm{n}=5)$; AT1 blocker $(\mathrm{n}=7)$; and statins $(\mathrm{n}=18)$. Aspirin was not stopped before the operation, and other agents were taken until the day before the operation.

For in vitro tension measurements, RA specimens were excised from the distal or proximal ends of RA grafts; placed in ice-cold modified Krebs-Henseleit buffer comprising $119 \mathrm{mmol} / \mathrm{L} \mathrm{NaCl}, 4.7 \mathrm{mmol} / \mathrm{L} \mathrm{KCl}$, $1.2 \mathrm{mmol} / \mathrm{L} \mathrm{KK_{2 }} \mathrm{PO}_{4}, 1.5 \mathrm{mmol} / \mathrm{L} \mathrm{CaCl} 2,25 \mathrm{mmol} / \mathrm{L} \mathrm{NaHCO}$, and $11 \mathrm{mmol} / \mathrm{L}$ glucose, which had been aerated with a mixed gas consisting of $95 \% \mathrm{O}_{2}$ and $5 \% \mathrm{CO}_{2}$; and transported to the laboratory.

RAs that exhibited vasospasm during harvest by using electric cautery in a semiskeletonized fashion were used for measurements of in situ free blood flow before and after intraradial administration of antispasmodics. Each artery was allowed to bleed freely for 10 seconds for the determination of free blood flow. Then patients received intraradial injection of $1.5 \mathrm{~mL}$ of either fasudil $(1.5 \mathrm{mg} / \mathrm{mL})$ or VG $(30 \mu \mathrm{mol} / \mathrm{L}$ verapamil and $30 \mu \mathrm{mol} / \mathrm{L}$ glyceryl trinitrate) ${ }^{8}$ in Ringer's solution (supplemented with $0.67 \mathrm{mmol} / \mathrm{L} \mathrm{NaHCO}_{3}$ and $1.67 \mathrm{U} / \mathrm{mL}$ heparin) from the distal end of the bilateral RAs, and the distal end of the bilateral RAs was occluded with a hemoclip. The right and left RAs were randomly assigned to fasudil or VG, and fasudil or VG was infused into the RAs in random order. Maximal dilatation of the RA was observed by 5 minutes after infusion of fasudil or VG solution. At 5 minutes, bilateral RAs were declipped, and in situ free blood flow was determined again. For the determination of phosphorylation of MYPT1 and MLC, the specimens were excised from the distal ends of the RA before and after antispasmodic injection and were rapidly frozen in an acetone dry ice slurry containing $20 \mathrm{mmol} / \mathrm{L}$ dithiothreitol and $10 \%$ trichloroacetic acid. ${ }^{22}$ As stated in the Results section, VG turned out to be less effective at increasing free blood flow than fasudil. Therefore VG-treated RAs subsequently received intraradial application of fasudil before use as grafts.

\section{Determinations of Tension and Phosphorylation of MYPT1 and MLC}

RAs were dissected free of adhering connective tissues and fat, cut into rings of about 4 to $5 \mathrm{~mm}$ in length (total of 61 rings from 15 patients), and denuded of endothelium by means of gentle rubbing of the internal surface with a wooden stick. The rings were mounted under $1 \mathrm{~g}$ of resting tension, and the isometric tension was determined as described previously. ${ }^{22}$ The tension during the measurement was expressed as the percentage of the maximum force during $\mathrm{KCl}$ precontraction. ${ }^{22}$

Isometrically contracted RA rings were rapidly frozen in acetone dry ice slurry and washed, as described previously. ${ }^{22}$ Fixed tissues were homogenized in a homogenization buffer of the following composition: $20 \mathrm{mmol} / \mathrm{L}$ Tris/HCl (pH 7.5); $100 \mathrm{mmol} / \mathrm{L} \mathrm{NaF} ; 1 \mathrm{mmol} / \mathrm{L} \mathrm{Na}_{3} \mathrm{VO}_{4} ; 1 \mathrm{mmol} / \mathrm{L}$ phenylmethylsulfonyl fluoride; $0.1 \%$ sodium dodecylsulfate; $2 \mathrm{mmol} / \mathrm{L}$ ethyleneglycol-bis-( $\beta$-aminoethylether)-N,N, $\mathrm{N}^{\prime}, \mathrm{N}^{\prime}$-tetraacetic acid; $0.5 \%$ NP-40; and $20 \mu \mathrm{g} / \mathrm{ml}$ each of leupeptin and aprotinin. The samples (80 $\mu \mathrm{g}$ of protein) were separated on $8 \%$ (for MYPT1) or $15 \%$ (for MLC) sodium dodecylsulfate-polyacrylamide gel electrophoresis, followed by Western blot analysis with either phospho( $\operatorname{Thr}^{853}$ )-MYPT1- or phospho(Ser $\left.{ }^{19}\right)$-MLC-specific antibodies. The same samples were also analyzed by means of Western blotting with antibodies that recognize both phospho- and non-phospho-proteins. The amounts of 
phospho(Thr $\left.{ }^{853}\right)$-MYPT1 and phospho(Ser $\left.{ }^{19}\right)$-MLC quantified by means of densitometry were normalized for total amounts of MYPT1 and MLC, respectively, in each sample, and the normalized quantified data of the phospho-proteins were expressed as multiples over the values in the nontreated RA ring and nonspastic RA, which are expressed as 1.0.

\section{Statistical Analysis}

All data are shown as means \pm standard errors of the mean. One-way or 2-way analysis of variance followed by the Bonferonni post test or paired or nonpaired 2-tailed Student's $t$ test was performed to determine the statistical significance of differences between mean values.

\section{RESULTS}

\section{Fasudil Inhibits Agonist-Induced RA Contraction}

We studied the effect of fasudil on agonist-induced isometric contraction of RA rings. The representative timecourse study shows that fasudil inhibited NA-induced contraction in a dose-dependent manner when fasudil wascumulatively added to rings precontracted by NA (Figure 1, $A$ ). Fasudil also inhibited 5-HT-induced contraction in a dose-dependent manner (Figure 1, B). Fasudil displayed similar inhibitory potencies for both receptor agonists (inhibitory concentration of $50 \%$ values of $2.94 \pm 0.34$ $\mu \mathrm{mol} / \mathrm{L}$ and $3.00 \pm 0.26 \mu \mathrm{mol} / \mathrm{L}$ for NA and $5-\mathrm{HT}$, respectively). Fasudil at $30 \mu \mathrm{mol} / \mathrm{L}$ suppressed NA- and 5-HTinduced contraction of all tested RA preparations from different patients by more than $90 \%$ (Figure 1, B).

We compared the efficacies of fasudil $(30 \mu \mathrm{mol} / \mathrm{L})$ and other antispasmodics (ie, $\mathrm{VG}^{6-8}$ the nonselective phosphodiesterase inhibitor papaverine, ${ }^{6,11}$ and the phosphodiesterase III-specific inhibitor olprinone ${ }^{9,10}$ ) in inhibiting NA- and 5-HT-induced contraction. Fasudil markedly inhibited NA- and 5-HT-induced contraction $(9.2 \% \pm$ $6.4 \%$ and $8.9 \% \pm 4.9 \%$ of maximal tension elicited by NA and 5-HT, respectively), which was comparable with the effects of VG $(8.5 \% \pm 5.1 \%$ and $8.5 \% \pm 4.8 \%$ of maximal tension elicited by NA and 5-HT, respectively; Figure $2, A)$. Olprinone $(30 \mu \mathrm{mol} / \mathrm{L})$ and papaverine (30 $\mu \mathrm{mol} / \mathrm{L}$ ) considerably inhibited contraction induced by NA and 5 -HT $(22.2 \% \pm 4.2 \%$ and $28.1 \% \pm 2.5 \%$ after olprinone treatment and $28.1 \% \pm 3.9 \%$ and $27.1 \% \pm$ $3.6 \%$ after papaverine treatment, respectively), although the extents of inhibition were less than those seen with fasudil and VG (Figure 2, A). Thus these antispasmodics all exhibited substantial efficacy in inhibiting receptor agonist-induced contraction of RAs.

\section{Fasudil Inhibits NA-Induced MYPT1 Phosphorylation}

We studied the effects of fasudil and VG on NA-induced phosphorylation of MYPT1 at the inhibitory phosphorylation site $\mathrm{Thr}^{853}$ in RA rings. NA induced about a 4-fold increase in MYPT1 phosphorylation (Figure 2,B), indicating that NA induced inhibition of MLCP. Pretreatment of RA rings with fasudil abolished NA-induced MYPT1
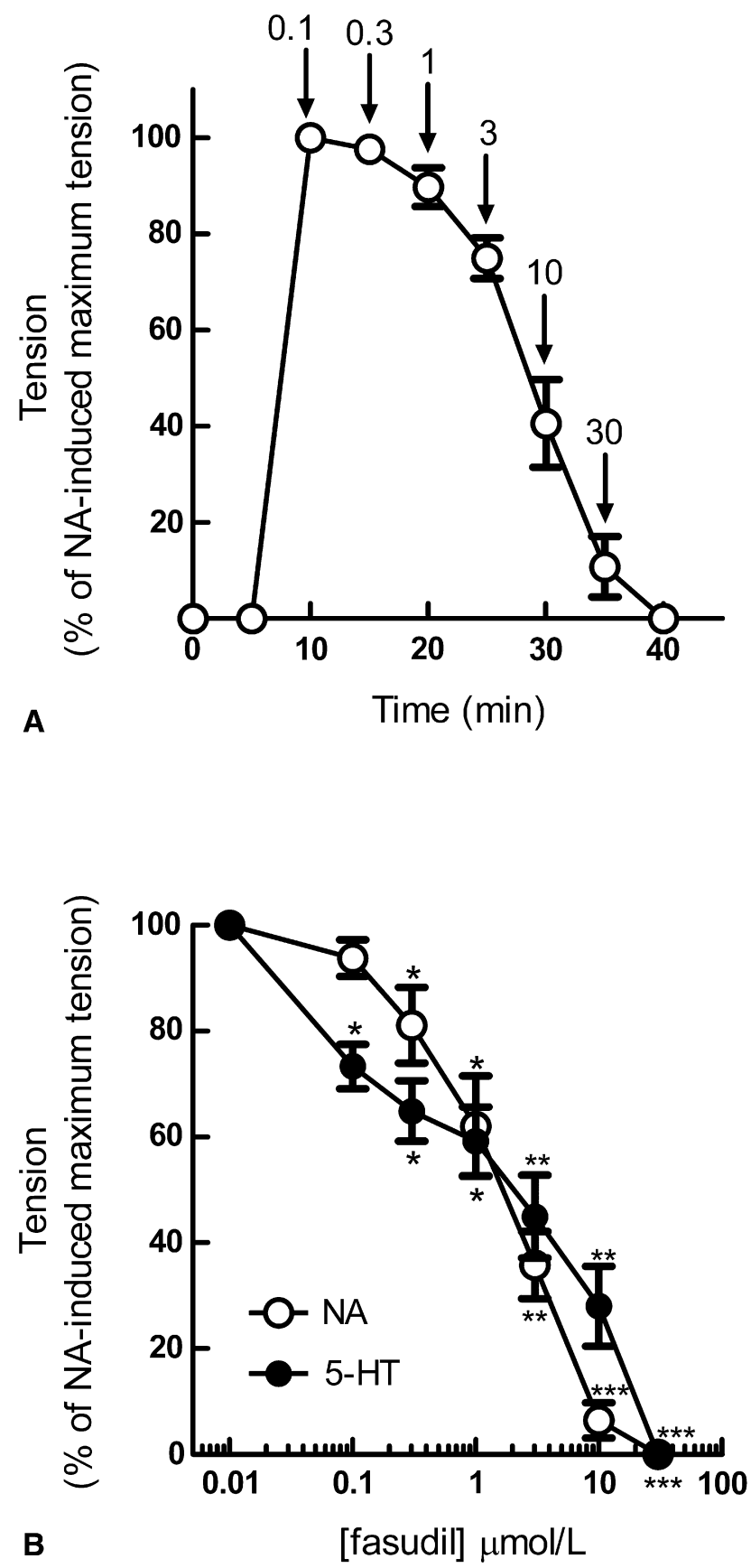

FIGURE 1. Fasudil inhibits receptor agonist-induced contraction of radial artery rings in a dose-dependent manner. A, Dose-dependent inhibition by fasudil of noradrenaline (NA)-elicited isometric contraction of ring preparations of radial arteries from one patient. At 10 minutes after the addition of NA $(1 \mu \mathrm{mol} / \mathrm{L})$, fasudil was added in a cumulative fashion (0.1-30 $\mu \mathrm{mol} / \mathrm{L})$ at the indicated time points. The values are presented as means \pm standard errors of the mean of the data from 3 rings. B, Dose-dependent inhibition of NA- and 5-hydroxytryptamine (5-HT)-induced contraction by fasudil. Isometric tension of human radial artery rings from 3 patients stimulated with NA $(1 \mu \mathrm{mol} / \mathrm{L})$ and $5-\mathrm{HT}(1 \mu \mathrm{mol} / \mathrm{L})$ is shown. Fasudil was added to radial artery rings 10 minutes after agonist stimulation. $* P<.05, * * P<.01$, and $* * * P<.001$ compared with nontreated radial arteries. 

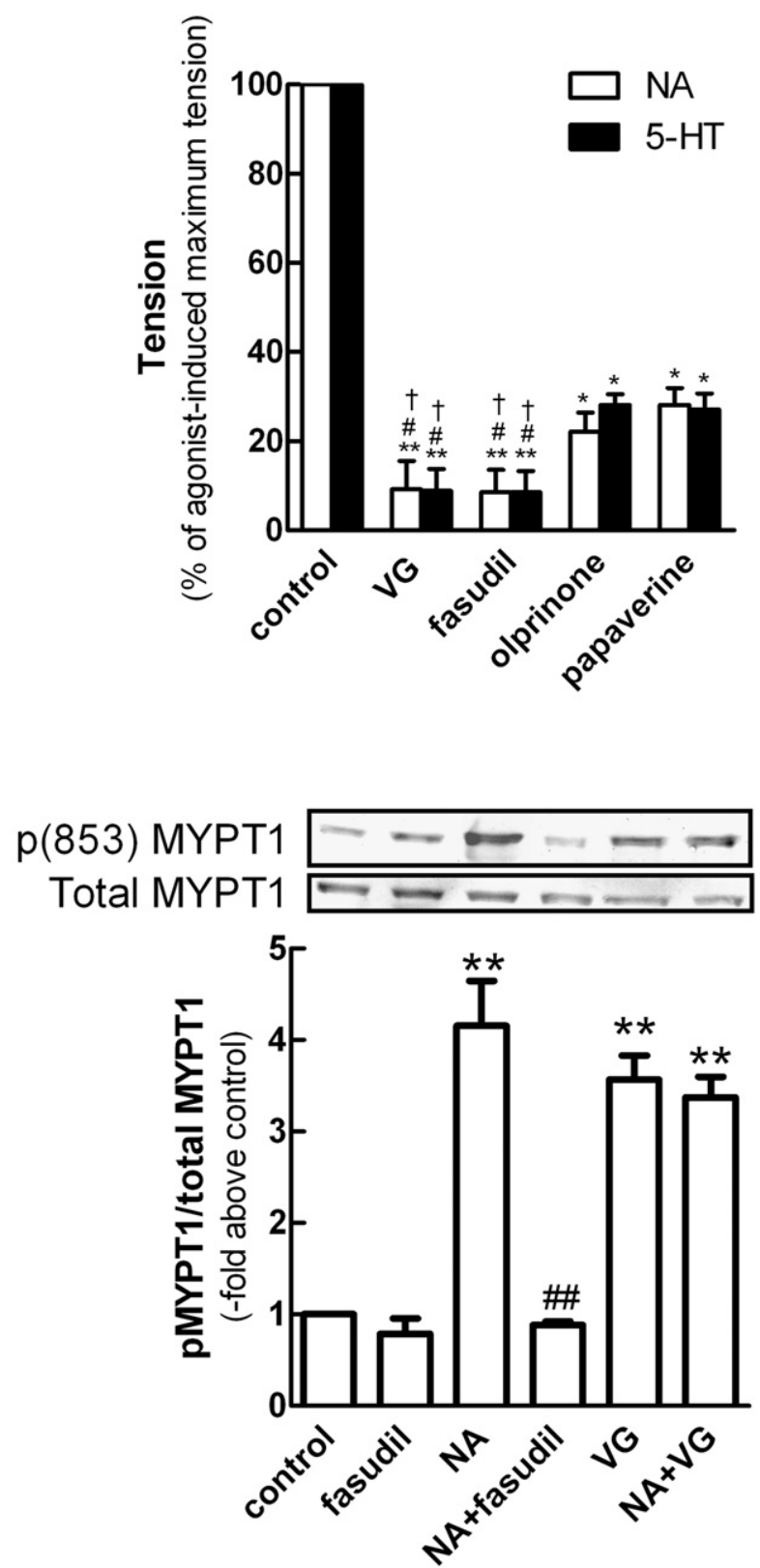

B

FIGURE 2. Fasudil-induced inhibition of contraction is accompanied by inhibition of myosin phosphatase targeting subunit 1 (MYPT1) phosphorylation in radial artery rings. A, Inhibition of noradrenaline (NA)and 5-hydroxytryptamine (5-HT)-induced contraction by various antispasmodics. At 10 minutes after the addition of NA $(1 \mu \mathrm{mol} / \mathrm{L})$ or $5-\mathrm{HT}$ $(1 \mu \mathrm{mol} / \mathrm{L})$ to radial artery rings, relaxation was induced by adding either fasudil, verapamil-glyceryl trinitrate $(V G)$, olprinone, or papaverine at a concentration of $30 \mu \mathrm{mol} / \mathrm{L}$. The maximal relaxation responses were determined after the addition of the drugs. $* P<.05$ and $* * P<.01$ compared with nontreated radial arteries. $\# P<.05$ compared with olprinone-treated radial arteries. $\dagger P<.05$ compared with papaverine-treated radial arteries. B, Inhibition by fasudil and VG of NA-induced phosphorylation of MYPT1. Isometrically contracted or resting radial artery rings were rapidly frozen at 10 minutes after the addition of fasudil or VG $(\mathrm{n}=3)$. ** $P<.01$ compared with control. \#\#P<.01 compared with fasudil-treated radial arteries. phosphorylation, suggesting that NA stimulates MYPT1 phosphorylation through ROCK (Figure 2, B). Paradoxically, VG alone induced an increase in MYPT1 phosphorylation. Pretreatment of RA rings with VG did not alter NA-induced MYPT1 phosphorylation (Figure 2, B). These observations suggest that fasudil, but not $\mathrm{VG}$, inhibits NA-induced contraction through inhibition of ROCK.

\section{Intraluminal Application of Fasudil in Spastic RAs Increases In Situ Free Blood Flow With Inhibition of Phosphorylation of MYPT1 and MLC}

In semiskeletonized spastic RAs intraluminal application of either fasudil or VG significantly increased in situ free blood flow (Figure 3, A). The increase in free blood flow before and after intraradial injections of antispasmodics was greater in fasudil-treated RAs compared with that seen in VG-treated RAs (Figure 3,B). The phosphorylation level of MYPT1 in semiskeletonized spastic RAs was approximately 4-fold increased compared with that seen in pedicled nonspastic RAs (Figure 4, A). Intraluminal injection of
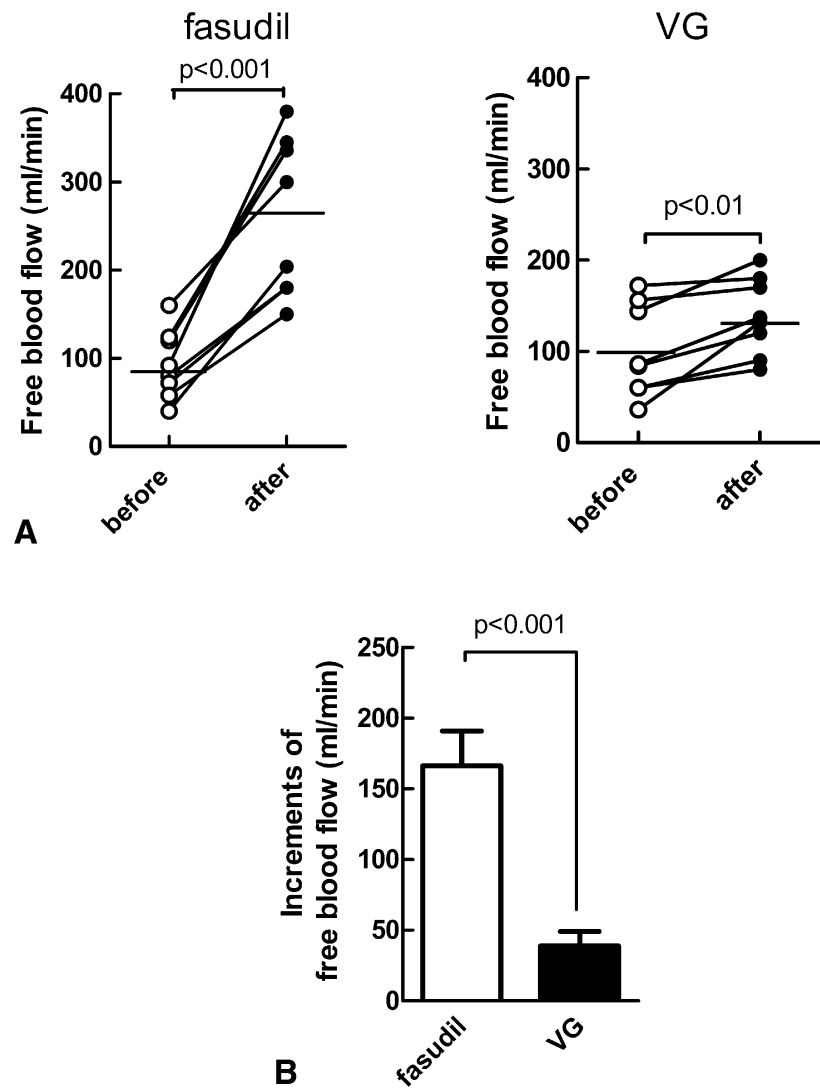

FIGURE 3. Intraradial administration of fasudil more effectively increases in situ free blood flow in spastic radial arteries than verapamil-glyceryl trinitrate $(V G)$. A, In situ free blood flow in bilateral semiskeletonized spastic radial arteries before and after infusion of either fasudil (left) or VG (right) in 8 patients is shown. B, The changes in in situ free blood flow before and after infusion of antispasmodics in bilateral radial arteries of 8 patients. 

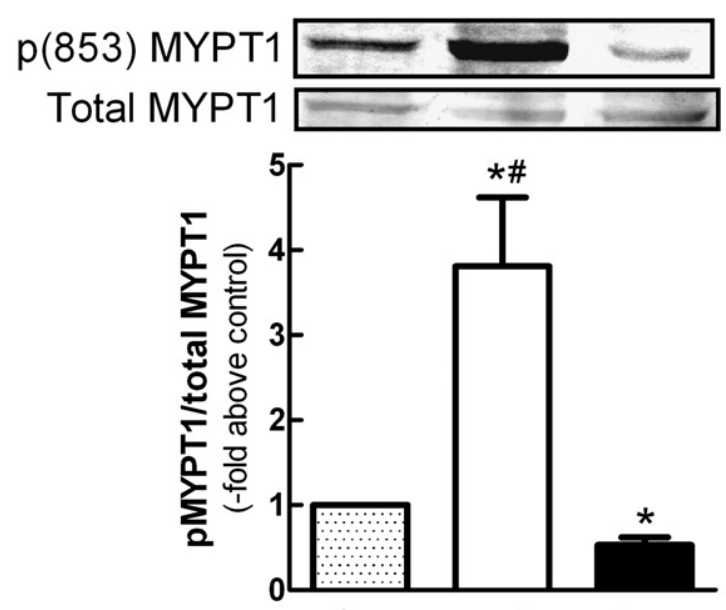

A

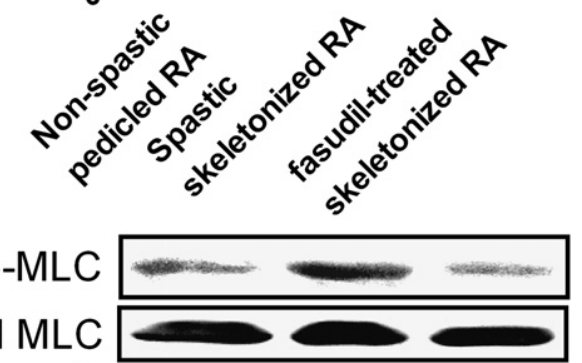

B

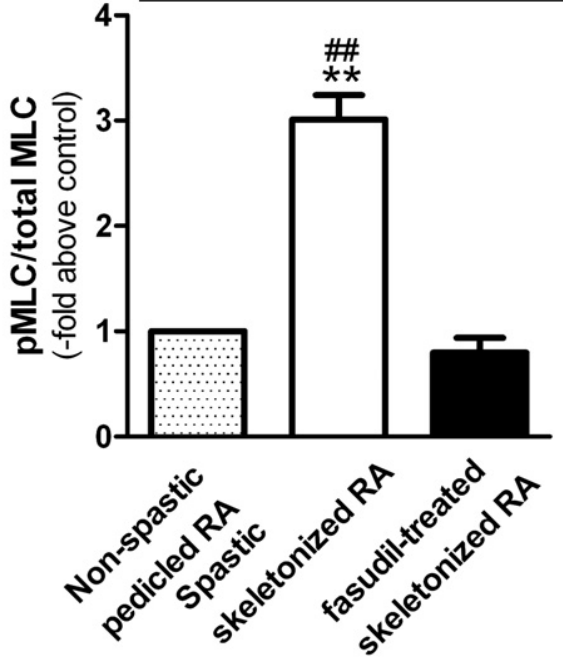

FIGURE 4. Intraradial administration of fasudil significantly inhibits phosphorylation of myosin phosphatase targeting subunit 1 (MYPT1; A) and myosin light chain $(M L C ; \mathrm{B})$ in skeletonization-induced spastic radial arteries (RAs). Distal segments of pedicled nonspastic RAs and subsequently skeletonized spastic RAs before and after fasudil infusion were excised, rapidly frozen, and analyzed for phosphorylation of MYPT (A) or MLC (B) by means of Western blotting. The data are derived from 3 to 5 values from different patients. $* P<.05$ and $* * P<.01$ compared with pedicled RAs. $\# P<.05$ and \#\#P<.01 compared with fasudil-treated RAs.

fasudil reduced MYPT1 phosphorylation to less than the level of nonspastic RAs. Consistent with the results of MYPT1 phosphorylation, the phosphorylation level of MLC was 3-fold increased in spastic RAs compared with that seen in nonspastic RAs, and fasudil injection decreased MLC phosphorylation to the level seen in nonspastic RAs
(Figure $4, B$ ). Intraradial application of fasudil or VG did not acutely affect hemodynamic parameters, including systemic blood pressure, heart rate, and cardiac output. Any patient who underwent CABG by using fasudil-treated RA grafts in the present study did not exhibit signs of myocardial ischemia or low cardiac output during the perioperative time period up to 72 hours after surgical intervention. These observations suggest that ROCK-dependent inhibition of MLCP and the resultant increase in MLC phosphorylation are involved in RA spasm and that fasudil effectively deinhibits MLCP, decreases MLC phosphorylation, and thereby relieves RA spasm.

\section{DISCUSSION}

The following findings in the present study suggest the usefulness of the ROCK inhibitor fasudil as a novel antispasmodic agent. First, in isolated RA ring preparations fasudil strongly inhibited contraction elicited by the 2 major vasoactive receptor agonists NA and 5-HT with abolition of agonist-induced stimulation of MYPT1 phosphorylation, indicating that ROCK-mediated inhibition of MLCP plays a crucial role in vasoconstrictor-induced contraction in this artery. Second, semiskeletonized spastic RAs exhibited enhanced ROCK activity compared with nonspastic RAs. Third, intraradial administration of fasudil, but not VG, inhibited MYPT1 phosphorylation in spastic RAs and effectively relieved vasospasm in situ. These observations document that increased ROCK activity is critically involved in RA spasm in CABG surgery and that fasudil is a quite effective antispasmodic that acts through the novel mechanism of ROCK inhibition.

Vasospasm in the RA is reported to occur in up to $10 \%$ of cases perioperatively. ${ }^{23}$ Arterial spasm during graft harvesting is suggested to be caused by various physical stimuli, such as mechanical stress resulting from the surgical procedure, temperature stress produced by cooling, nervous stimuli, and vasoactive humoral mediators. ${ }^{1,6-8}$ The spastic property of the thick muscular wall and enhanced reactivity to vasoactive mediators are implicated in higher susceptibility to vasospasm of the RA. ${ }^{1,6}$ However, the exact mechanisms of perioperative RA spasm remain unknown. Recently, Rho- and ROCK-dependent inhibition of MLCP has been shown to serve an essential role in vasoconstriction in various vascular beds. $^{12,13}$ Our data showed that on vasoactive receptor agonist stimulation, ROCK-dependent MLCP inhibition operates and substantially contributes to contraction in nonspastic RAs (Figures 1 and 2). Importantly, the present study showed that skeletonization-induced acute RA spasm was accompanied by exaggerated MLCP inhibition and consequent increased levels of MLC phosphorylation (Figure 4), suggesting that the ROCK-MLCP pathway is causally related to the pathogenesis of RA spasm.

The in vivo effectiveness of fasudil for RA spasm during CABG surgery has been previously totally unknown. The 
present study demonstrated for the first time that intraluminal administration of fasudil markedly increased in situ free blood flow in spastic RAs and that the effect of fasudil was much greater than that of the current standard antispasmodic VG (Figure 3). Our data suggested that the antispastic effect of fasudil was due to blockade of ROCK because fasudil injection abolished an increase in MYPT1 phosphorylation at the ROCK phosphorylation site $\mathrm{Thr}^{853}$ and, consequently, MLC phosphorylation (Figure 4). It should be noted that the mechanisms of fasudil action are different from those of other antispasmodic agents; VG by itself rather increased MYPT1 phosphorylation in nonspastic RAs and did not affect NA-induced MYPT1 phosphorylation (Figure 2, B). Glycerol trinitrate, a component of VG mixture, releases nitric oxide and resultantly increases levels of intracellular cyclic guanosine monophosphate. ${ }^{12}$ Cyclic guanosine monophosphate was previously shown to inhibit Rho. However, the cyclic nucleotide also has inhibitory actions on $\mathrm{Ca}^{2+}$ channels and phospholipase $\mathrm{C}{ }^{9}$ Another component of VG, verapamil, is a $\mathrm{Ca}^{2+}$ channel antagonist. ${ }^{6-8}$ Therefore it is likely that VG inhibits NAinduced contraction through a mechanism independent of ROCK-mediated MLCP inhibition. It remains to be clarified how VG induced MYPT1 phosphorylation.

Previous studies implicated augmented ROCK activity in the pathogenesis of various types of vasospasm; experimental vasospasm of coronary and cerebral vessels in animals was accompanied by an increased vascular activity of ROCK and enhanced MLCP inhibition. ${ }^{14-17}$ In patients administration of fasudil alleviated vasospasm after subarachnoid hemorrhage and coronary vasospasm. ${ }^{15-17}$ In a small number of patients shortly after CABG, intracoronary infusion of fasudil relieved severe coronary vasospasm. ${ }^{18}$ These observations suggest that augmented MLCP inhibition might be involved in different types of vasospasm through increased MLC phosphorylation.

ROCK is also implicated in endothelial dysfunction, which includes inhibition of the nitric oxide-synthesizing enzyme exhaled nitric oxide synthase, upregulation of leukocyte adhesion molecule expression, and upregulation of proinflammatory cytokine expression. ${ }^{14}$ Therefore fasudil might protect the endothelium from damage, leading to the prevention of intimal proliferation, acceleration of atherosclerosis, and thrombosis, which likely improves the long-term patency of grafts.

In the present study intraradial administration of fasudil did not affect the hemodynamic parameters or cause other adverse effects, which is consistent with the reports in patients with cerebral vasospasm after subarachnoid hemorrhage who received fasudil administration. ${ }^{24}$ In the present study the RA conduits remained dilated during the operation after short-term fasudil treatment. None of the 8 patients who had undergone grafting of fasudiltreated RAs exhibited myocardial ischemia or cardiac pump failure. Clinical trials of fasudil as an antispasmodic agent need to be expanded for its further clinical validation.

Adequate use of antispastic agents is crucial to maintaining graft patency and avoiding myocardial ischemia perioperatively. Taking into consideration that fasudil was a much more powerful antispasmodic than VG, which was reported to be the optimum agent, it is concluded that fasudil is a very effective new generation of antispasmodic that acts through a distinct mechanism.

\section{References}

1. Kobayashi J. Radial artery as a graft for coronary artery bypass grafting. Circ J. 2009;73:1178-83.

2. Buxton BF, Hayward PA, Newcomb AE, Moten S, Seevanayagam S, Gordon I. Choice of conduits for coronary artery bypass grafting: craft or science? Eur J Cardiothorac Surg. 2009;35:658-70.

3. Collins P, Webb CM, Chong CF, Moat NE. Radial Artery Versus Saphenous Vein Patency (RSVP) Trial Investigators. Radial artery versus saphenous vein patency randomized trial: five-year angiographic follow-up. Circulation. 2008;117: 2859-64.

4. Hayward PA, Gordon IR, Hare DL, Matalanis G, Horrigan ML, Rosalion A, et al. Comparable patencies of the radial artery and right internal thoracic artery or saphenous vein beyond 5 years: results from the Radial Artery Patency and Clinical Outcomes trial. J Thorac Cardiovasc Surg. 2010;139:60-5.

5. He GW. Arterial grafts for coronary artery bypass grafting: biological characteristics, functional classification, and clinical choice. Ann Thorac Surg. 1999;67: 277-84.

6. He GW. Verapamil plus nitroglycerin solution maximally preserves endothelial function of the radial artery: comparison with papaverine solution. J Thorac Cardiovasc Surg. 1998;115:1321-7.

7. He GW, Yang CQ. Use of verapamil and nitroglycerin solution in preparation of radial artery for coronary grafting. Ann Thorac Surg. 1996;61:610-4.

8. He GW, Fan L, Furnary A, Yang Q. A new antispastic solution for arterial grafting: Nicardipine and nitroglycerin cocktail in preparation of internal thoracic and radial arteries for coronary surgery. J Thorac Cardiovasc Surg. 2008;136:673-80.

9. He GW, Yang CQ. Vasorelaxant effect of phosphodiesterase-inhibitor milrinone in the human radial artery used as coronary bypass graft. $J$ Thorac Cardiovasc Surg. 2000;119:1039-45.

10. Adachi H, Kamata S, Kodama K, Negakura T. Vasorelaxant effect of a phosphodiesterase 3 inhibitor, olprinone, on isolated human radial artery. Eur J Pharmacol. 2000;396:43-7.

11. Mussa S, Guzik TJ, Black E, Dipp MA, Channon KM, Taggart DP. Comparative efficacies and durations of action of phenoxybenzamine, verapamil/nitroglycerin solution, and papaverine as topical antispasmodics for radial artery coronary bypass grafting. J Thorac Cardiovasc Surg. 2003;126:1798-805.

12. Somlyo AP, Somlyo AV. $\mathrm{Ca}^{2+}$ sensitivity of smooth muscle and nonmuscle myosin II: modulated by G proteins, kinases, and myosin phosphatase. Physiol Rev. 2003;83:1325-58.

13. Takuwa Y, Yoshioka K, Takuwa N, Wang Y, Azam MA, Sugimoto N. Calciumdependent regulation of Rho and myosin phosphatase in vascular smooth muscle. Biomed Res. 2005;16:13-21.

14. Kobayashi M, Tanoue Y, Eto M, Baba H, Kimura S, Oda S, et al. A Rho-kinase inhibitor improves cardiac function after 24-hour heart preservation. $J$ Thorac Cardiovasc Surg. 2008;136:1586-92.

15. Shibuya M, Suzuki Y, Sugita K, Saito I, Sasaki T, Takakura K, et al. Effect of AT877 on cerebral vasospasm after aneurismal subarachnoid hemorrhage. Results of a prospective placebo-controlled double-blind trial. J Neurosurg. 1992; 76:571-7.

16. Masumoto A, Mohri M, Shimokawa H, Urakami L, Usui M, Takeshita A. Suppression of coronary artery spasm by the Rho-kinase inhibitor fasudil in patients with vasospastic angina. Circulation. 2002;105:1545-7.

17. Mohri M, Shimokawa H, Hirakawa Y, Matsumoto A, Takeshita A. Rho-kinase inhibition with intracoronary fasudil prevents myocardial ischemia in patients with coronary microvascular spasm. J Am Coll Cardiol. 2003;41:15-9.

18. Inokuchi K, Ito A, Fukumoto Y, Matoba T, Shiose A, Nishida T, et al. Usefulness of fasudil, a Rho-kinase inhibitor, to treat intractable severe coronary spasm after coronary artery bypass surgery. J Cardiovasc Pharmacol. 2004;44:275-7. 
19. Shackcloth MJ, Conant AR, Thekkudan J, Ghotkar S, Simpson AWM, Dihmis WC. Attenuation of receptor-dependent and -independent vasoconstriction in the human radial artery. Eur J Cardiothorac Surg. 2008;34:839-44.

20. Olcay MD, Ercan O, Ocal B, Ihsan B, Nedim D, Ahmet P. Rho-kinase inhibitors Y-27632 and fasudil prevent agonist-induced vasospasm in human radial artery. Can J Physiol Pharmacol. 2009;87:595-601.

21. Xia K, Wang L, Ding R, Yang X. RhoA/ROK pathway related to the mechanism of higher susceptibility to spasm in RA than in IMA. J Card Surg. 2009;24: 766-71.
22. Azam MA, Yoshioka K, Ohkura S, Takuwa N, Sugimoto N, Sato K, et al. $\mathrm{Ca}^{2+}$ independent, inhibitory effects of cyclic adenosine 5'-monophosphate on $\mathrm{Ca}^{2+}$ regulation of phosphoinositide 3-kinase c2alpha, Rho, and myosin phosphatase in vascular smooth muscle. J Pharmacol Exp Ther. 2007;320:907-16.

23. Mussa S, Choudhary BP, Taggart DP. Radial artery conduits for coronary artery bypass grafting: current perspective. J Thorac Cardiovasc Surg. 2005;129:250-3.

24. Vicari RM, Chaitman B, Keefe D, Smith WB, Chrysant SG, Tonkon MJ, et al. Efficacy and safety of fasudil in patients with stable angina: a double-blind, placebo-controlled, phase 2 trial. J Am Coll Cardiol. 2005;46:1803-11. 